\title{
Dipole scattering in polarization induced two-dimensional electron gases
}

\author{
Debdeep Jena *, Arthur C. Gossard, Umesh. K. Mishra \\ Department of Electrical and Computer Engineering, University of California, Santa Barbara, \\ CA 93106
}

\begin{abstract}
Unusually large spontaneous and piezoelectric fields in the III-V nitrides have led to the making of an entirely new class of two-dimensional electron gas. Fluctuation from a perfectly periodic binary structure in highly polar semiconductor alloys present the same physical situation as a random distribution of microscopic dipoles. The excess dipole distribution in the barrier layers is evaluated by a method similar to the virtual crystal approximation. It is shown that the mobility of electrons in the two-dimensional electron gas formed in highly polar heterostructures is intrinsically limited by scattering from such dipoles.
\end{abstract}

*Electronic Mail: djena@indy.ece.ucsb.edu 


\section{INTRODUCTION}

The last decade has witnessed a formulation of the microscopic quantum theory of polarization based on a rigorous definition of polarization in a periodic system [Ref. 1-3]. The study of polarization and its effects was limited to ferroelectrics before the advent of III-V nitride semiconductors. The spontaneous and piezoelectric polarization fields of this class of semiconductors is unusually high compared to other III-Vs; in fact, it has given birth to a new frontier of semiconductor physics. The wide band gap and strong polarization fields have found wide application in high power, high speed electronic and optical devices [Ref. 4,5]. A large amount of effort has been devoted to the fabrication and study of high power AlGaN/GaN heterostructure high electron mobility transistors (HEMTs) [Ref. 6]. Of chief importance in such devices is the electron mobility in the two-dimensional electron gas (2DEG) formed at the heterointerface. The strong polarization of the III-V nitride heterostructures makes a clear analysis of the effect of microscopic polarization on mobility of considerable interest to device designers and theorists alike.

At high temperatures $(T \geq 100 K)$ polar optical phonon scattering is the largest scattering mechanism; the effects of the other scattering mechanisms become dominant only at low temperatures. The set of scattering mechanisms needed to understand the low temperature 2DEG mobility of the AlGaAs/GaAs and Si-metal oxide field effect transistor (MOSFET) is fairly complete. Theoretical mobility calculations show very good agreement with experimentally observed values [Ref 7,8]. However, the 2DEGs in III-V nitride MDHs have a fundamentally new origin. The 2DEG in such heterostructures can be entirely polarization induced, as opposed to by remote doping as in AlGaAs/GaAs MDHs and gate-induced inversion in Si-MOSFETs. In fact the spontaneous and piezoelectric polarization is large enough to produce 2DEGs without intentionally doping the barrier, leading to the novel concept of piezoelectric doping in such systems [Ref. 9,10]. The term 'modulation doped hetersostructures' (MDHs) is somewhat erroneous in such devices, so we prefer to call it the more general HEMTs.

Previously published calculations of mobilities in AlGaN/GaN 2DEGs have restricted their analysis to the set of scattering mechanisms that exists for AlGaAs/GaAs 2DEGs [Ref. 11,12]. This existing set of well understood scattering mechanisms is insufficient for polarization induced 2DEGs. It needs to be expanded by inclusion of the effects of the different origins of polarization induced 2DEGs. This work is directed towards this extention for the fundamentally new 2DEGs. In section II, we theoretically derive the transport scattering rates for 2DEG electrons due to dipoles. In section III, the results are applied to study the III-V nitride heterostructure 2DEG mobilities. In Section IV, we conclude with a discussion of the results, evaluation of the implications of the newly identified scattering mechanism and suggestion of a method to reduce it's effect.

\section{THEORY}

Scattering by dipoles and their effects on electron transport in bulk semiconductor samples has been studied, albeit not extensively owing to it's insignificance in the non-polar Si and relatively weakly polar GaAs material systems [Ref. 13,14]. However, the effect of dipole scattering on 2DEG electron transport has not been studied to the best of our knowledge. 
We derive the scattering rate due to dipoles for a semiconductor two dimensional electron gas.

We consider the 2DEG to be perfect (i.e. ,the extent along the $z$ direction to be zero) for our derivation. Extention to the more physical case of a 2DEG with finite extent along the growth direction involves incorporation of the relevant form factors.

Figure[1] shows the model for the system under consideration. The dipole charges are separated from each other by distance $d_{0}$, and the center is a distance $z$ from the plane containing the 2DEG. Spontaneous and piezoelectric polarization fields $\left(\mathbf{P}_{\mathbf{s p}}\right.$ and $\mathbf{P}_{\mathbf{p z}}$ respectively) in wurtzite AlGaN/GaN is directed perpendicular to the 2DEG plane [Ref. 15]. We choose the direction of the dipole to be perpendicular to the 2DEG plane to reflect this.

The unscreened Coulomb potential at the origin due the dipole is written as

$$
V_{u n s}(r, z)=\frac{e}{4 \pi \epsilon_{0} \epsilon_{b}} \cdot\left[\frac{e}{\sqrt{r^{2}+\left(z-\frac{d_{0}}{2}\right)^{2}}}-\frac{e}{\sqrt{r^{2}+\left(z+\frac{d_{0}}{2}\right)^{2}}}\right] .
$$

Here $e$ is the electron charge, $\epsilon_{0}$ is the permittivity of free space, $\epsilon_{b}$ is the dielectric constant of the subsrate semiconductor, and $r$ is the in-plane radius vector.

We evaluate the Fourier transform of this potential in the wavevector $(q)$ space $V_{\text {uns }}(q)=$ $\int V_{\text {uns }}(r) e^{i \mathbf{q} \cdot \mathbf{r}} d^{3} r$ to get

$$
V_{u n s}(q, z)=\frac{e^{2}}{2 \epsilon_{0} \epsilon_{b}} \cdot \frac{2 e^{-q z} \sinh \left(\frac{q d_{0}}{2}\right)}{q},
$$

where $q$ is the $x-y$ in-plane wavevector.

The Fourier component of screened and unscreened potentials are related for a degenerate 2DEG through the Thomas Fermi approximation of the Lindhard formula for the momentum dependent static dielectric constant [Ref. 16]. It is written as $\epsilon_{2 D}(q)=1+\frac{q_{T F}}{q}$, where $q_{T F}$ is the Thomas-Fermi wavevector, defined as $\frac{2}{a_{B}^{*}}, a_{B}^{*}$ being the effective Bohr radius in the semiconductor containing the 2DEG. A valley degeneracy of one and spin degeneracy of two is implied. The relation is

$$
V_{s c r}(q, z)=\frac{V_{u n s}(q, z)}{\epsilon_{2 D}(q)}=\frac{e^{2}}{2 \epsilon_{0} \epsilon_{b}} \cdot \frac{2 e^{-q z} \sinh \left(\frac{q d_{0}}{2}\right)}{q+q_{T F}} .
$$

This is the final screened potential experienced by an electron in the 2DEG due to a single dipole at a distance $z$ from the $2 \mathrm{DEG}$ plane.

Scattering rate from a state $\mid \mathbf{k}>$ to a state $\mid \mathbf{k}+\mathbf{q}>$ is now evaluated. Born approximation holds good for evaluation of matrix elements for dilute dipole concentrations. Transport scattering rate by a dilute perturbing potential in the Born approximation is written as [Ref. $17]$

$$
\frac{1}{\tau_{t r}^{2 D}}=n_{i m p}^{2 D} \frac{2 \pi}{\hbar} \int \frac{q^{2}}{2 k^{2}}\left|V_{s c r}^{t o t}(q)\right|^{2} \delta[E(k+q)-E(k)] \frac{d^{2} q}{(2 \pi)^{2}},
$$

where $n_{i m p}^{2 D}$ is the $2 \mathrm{D}$ impurity concentration, $k$ is the $2 \mathrm{D}$ electron wavevector before scattering, $E(k)$ and $E(k+q)$ are the electron energies before and after scattering, the $\delta$ function is a statement of the elastic nature of the scattering, and for our case, $n_{i m p}^{2 D}$ is 
the density of dipoles at each $\mathrm{Al}(\mathrm{Ga})$ plane, $n_{\text {dipole }}^{2 D} . V_{s c r}^{t o t}(q)$ is the total screened potential experienced by the 2DEG due to the entire distribution of dipoles.

Figure [2] illustrates the physical location of the dipoles in AlGaN/GaN HEMTs. Their origin is discussed in section III. Owing to the interface roughness, there are dipoles located at the interface too; however, their effect on the 2DEG mobility was found to be much less than the far denser distribution of dipoles in the barrier. We consider the 2DEG to be physically located at the centroid of the spatially extending quasi-2DEG for illustrating the role of dipoles. A Fang-Howard wavefunction approach would yield respective multiplicative form factors, and is a simple extention of the theory presented here.

The screened potential due to the distribution of dipoles in the barrier is hence given by a Fourier-weighted sum over all dipoles [Ref. 16]

$$
V_{s c r}^{t o t}(q)=\sum_{i} e^{i \mathbf{q} \cdot \mathbf{r}_{\mathbf{i}}} \frac{V_{u n s}\left(q, z_{i}\right)}{\epsilon_{2 D}(q)} .
$$

If we assume that the dipole distrubution on each $\mathrm{Al}(\mathrm{Ga})$ plane are completely uncorrelated, the cross terms arising in the sum cancel, and we are left with a sum over different planes. The complex exponential can then be factored out and therefore does not contribute to the matrix element. For a thick AlGaN barrier, this evaluates to

$$
V_{s c r}^{\text {tot }}(q)=\frac{e^{2}}{2 \epsilon_{0} \epsilon_{b}} \cdot \frac{2 e^{-q\left(z_{0}+c_{0}\right)}}{1-e^{-q c_{0}}} \cdot \frac{\sinh \left(\frac{q d_{0}}{2}\right)}{q+q_{T F}},
$$

where $z_{0}$ is the distance of the centroid of the 2DEG from the interface [Figure [2]], and $c_{0}$ is the separation of the planes containing the dipoles in the barrier.

For a degenerate gas as in a 2DEG, scattering takes place mainly among electrons with wavevectors near the Fermi wavevector $k_{F}=\sqrt{2 \pi n_{s}}$ where $n_{s}$ is the 2DEG sheet density of carriers. So the $k$ in the integral can be replaced by $k_{F}$. The elastic nature of the scattering

process leads to a relation $q=2 k_{F} \cdot \sin \left(\frac{\theta}{2}\right)$ where $\theta$ is the angle between the wavevectors $\mathbf{k}$ and $\mathbf{k}+\mathbf{q}$. Using these facts, the expression for scattering rate simplifies to

$$
\frac{1}{\tau_{\text {dipole }}^{2 D}}=n_{\text {dipole }}^{2 D} \frac{m^{*}}{2 \pi \hbar^{3} k_{F}^{3}} \int_{0}^{2 k_{F}}\left|V_{\text {scr }}^{\text {tot }}(q)\right|^{2} \frac{q^{2} d q}{\sqrt{1-\left(\frac{q}{2 k_{F}}\right)^{2}}} .
$$

Using the screened scattering potential of the distribution of dipoles developed in Equation [6] in Equation [7], we get the final scattering rate due to dipoles.

\section{APPLICATION TO III-V NITRIDE HEMTS}

We concentrate on $\mathrm{AlGaN} / \mathrm{GaN}$ heterostructures in our analysis. Recent work shows that the 2DEG in such heterostructures is created by the subtle interplay of spontaneous and piezoelectric polarization [Ref. 15,18,19]. The ab initio calculations of Bernardini et al show that the III-V nitrides have unusually large polarization coefficients (an order of magnitude larger than AlGaAs/GaAs systems) [Ref. 15]. The 2DEG in such heterostructures can be entirely polarization induced. In that sense, it is fundamentally different from the 2DEG in 
AlGaAs/GaAs heterostructures, which is got by modulation doping. It is argued that the mobility in such a 2DEG should be affected by the cause of its very existence.

For the perfectly periodic III-V nitride crystal, the microscopic picture of polarization is a dipole in each primitive cell aligned along the (0001) axis. The dipole moment $\mathbf{p}_{\mathbf{0}}=e \cdot d_{0}$ ( $d_{0}$ is the effective charge separation) is related to the macroscopic polarization $\mathbf{P}$ by the relation $\mathbf{P}=\mathbf{p}_{\mathbf{0}} / \Omega$, where $\Omega$ is the volume of the primitive cell [Ref. 20]. $\mathbf{P}$ is the total polarization, which includes the spontaneous and piezoelectric components.

$$
\mathbf{P}=\mathbf{P}_{\mathrm{sp}}+\mathbf{P}_{\mathrm{pz}}
$$

A perfect binary polar lattice thus has a periodically arranged array of dipoles with equal dipole moments. Such a periodic arrangement of similar dipoles has a characteristic wavevector, and hence does not contribute to the scattering matrix element.

However, the 2DEG in AlGaN/GaN heterostructures is confined by a barrier due to the undoped $A l_{x} G a_{1-x} N$ ternary alloy barrier. The alloy is a disordered system with $\mathrm{Al}$ and Ga atoms arranged in a random array such that the overall composition over any plane is constant over $\mathrm{Al}(\mathrm{Ga})$ planes. The difference in spontaneous and piezoelectric polarizations between AlN and GaN implies that we have a dipole moment of randomly fluctuating magnitude in the barrier. We adopt a method similar to the treatment of disordered alloys by virtual crystal approximation to treat dipoles in disordered polar semiconductor alloys.

We first arrive at the dipole moments in a primitive cell of coherently strained AlN and GaN binary wurtzite crystals. $\mathbf{P}_{\mathbf{s p}}$ and the piezoelectric constants for both the semiconductors were calculated by Bernardini et. al. in their recent paper [Ref. 15]. The piezoelectric field in a binary wurtzite primitive cell coherently strained to a $x-y$ lattice constant $a(x)$ from it's unstrained lattice constant $a_{0}$ and $c(x)$ from $c_{0}$ in the $z$ direction is given by the relation [Ref. 18]

$$
P_{p z}(x)=2 \cdot\left(\frac{a(x)-a_{0}}{a_{0}}\right) \cdot\left[e_{31}-e_{33} \frac{C_{13}}{C_{33}}\right],
$$

where $e_{31}$ and $e_{33}$ are the piezoelectric coefficients and $C_{13}$ and $C_{33}$ are the elastic constants of the crystal structure. The volume of the primitive hexagonal cell is

$$
\Omega(x)=\frac{\sqrt{3}}{2} c_{0}(x) \cdot a_{0}^{2}(x) .
$$

Thus the dipole moment in a strained binary crystal is given by

$$
p_{\text {dipole }}(x)=\left(P_{s p}+P_{p z}(x)\right) \cdot \Omega(x) .
$$

This dipole moment is calculated for both semiconductors as $p_{\text {dipole,AlN }}(x)$ and $p_{\text {dipole }, \text { GaN }}(x)$.

We model the disordered $A l_{x} G a_{1-x} N$ barrier as a perfect crystal superposed with a randomly fluctuating dipole moment at each primitive cell. Such a virtual crystal has a dipole moment of magnitude

$$
p_{\text {dipole }}(a v)=x \cdot p_{\text {dipole }, \text { AlN }}(x)+(1-x) \cdot p_{\text {dipole }, \text { GaN }}(x) .
$$

The deviation from the perfect virtual crystal at all $\mathrm{Al}$ sites is $(1-x) \cdot \Delta p_{\text {dipole }}$ where 


$$
\Delta p_{\text {dipole }}=p_{\text {dipole }, A l N}(x)-p_{\text {dipole, GaN }}(x) .
$$

The deviation at Ga sites is $(-x) \cdot \Delta p_{\text {dipole }}$. Since there are $x \mathrm{Al}$ sites and $(1-x)$ Ga sites on average on a $\mathrm{Al}(\mathrm{Ga})$ plane, the average randomly fluctuating dipole moment at each site is

$$
\delta p_{\text {dipole }}=e \cdot d_{0}=2 \cdot x \cdot(1-x) \cdot\left|\Delta p_{\text {dipole }}\right| .
$$

The absolute value is used in adding the dipole contributions since the direction of the dipole is immaterial in the scattering matrix element, which involves the square of the dipole potential. The number of such dipoles present on each $\mathrm{Al}(\mathrm{Ga})$ plane is given by

$$
n_{\text {dipole }}^{2 D}=\frac{1}{\frac{\sqrt{3}}{4} a_{0}^{2}(x)},
$$

where the in plane lattice constant $a_{0}(x)$ is interpolated for the alloy.

The polarization induced 2DEG sheet density is given by the difference in polarization at the interface as a function of alloy fraction $x$ [Ref. 18],

$$
n_{s}(x)=\left|P_{p z}\left(A l_{x} G a_{1-x} N\right)+P_{s p}\left(A l_{x} G a_{1-x} N\right)-P_{s p}(G a N)\right| .
$$

Note that the 2DEG concentration does not involve any modulation dopants; it is formed entirely to satisfy the discontinuity in the polarization fields at the interface.

\section{RESULTS AND DISCUSSION}

The mobility inhibited by dipole scattering alone $\mu_{\text {dipole }}^{2 D}=e \tau_{\text {dipole }}^{2 D} / m^{*}$ is evaluated for different alloy compositions. The results are plotted in Figure [3]. We also plot the polarization induced 2DEG sheet density in the same figure for easy comparision. In Figure [4], we plot the dipole scattering inhibited 2DEG sheet conductivity given by $G=e \mu_{\text {dipole }}^{2 D} n_{s}$, and finally, in Figure [5], we plot the dipole scattering limited mobility as a function of the 2DEG sheet density.

An expected increase in mobility with the increase in the binary nature of the alloy barrier is seen. The intrinsic low temperature mobility limit in the $x=0.1$ to $x=0.4$ range (which is typical of state of the art III-V nitride HEMTs) is in the $400,000 \mathrm{~cm}^{2} / V s$ to $200,000 \mathrm{~cm}^{2} / V s$ range. It is well worth noticing that this is much lower than the record low temperature mobilities $\left(\approx 10^{7} \mathrm{~cm}^{2} / V s\right)$ of AlGaAs/GaAs modulation doped heterostructures, and an order of magnitude higher than the record high mobilities in AlGaN/GaN HEMTs observed till date $\left(51,700 \mathrm{~cm}^{2} / \mathrm{Vs}\right)$ [Ref. 19,21]. It hints at some more severe scattering mechanism(s) that determine the low temperature mobility in the III-V nitrides.

Interface roughness was initially thought to be a mobility limiting mechanism owing to a) very high 2DEG sheet densities, and b) trying to fit experimental data to the existing set of scattering mechanisms for AlGaAs/GaAs MDHs [Ref. 12,22]. Recent experiments with double heterostructures point towards the contrary [Ref. 23]. The III-V nitrides is a fundamentally new system, and it is necessary that we address issues that make the material system so different from the AlGaAs/GaAs systems. 
Dislocation scattering was identified as becoming dominant at high dislocation densities, which result from the lattice mismatch of the epitaxial GaN layer with the present substrates of choice - SiC or Sapphire [Ref. 24]. The novel method of lateral epitaxial overgrowth (LEO) is a promising candidate for reducing the density of dislocations in the nitrides [Ref. 25]. The effects of dipole scattering will be the next hurdle to overcome in pushing the mobilities higher. Digital alloy growth is suggested as a technique to reduce the severity of dipole scattering. By growing either purely $\mathrm{Al}$ or purely Ga layers, we use periodicity to overcome the scattering originating from the random nature of the alloy. However, digital alloy growth suffers from interdiffusion of atoms in the growth process, so dipole scattering cannot be completely eliminated by this method.

\section{ACKNOWLEDGMENTS}

The authors acknowledge illuminating discussions with S. Keller, I. P. Smorchkova, and J. S. Speck. The authors are grateful to I. P. Smorchkova for sharing her data on the latest record high low temperature mobilities in AlGaN/GaN 2DEGs. 


\section{REFERENCES}

[1] R. D. King-Smith and D. Vanderbilt, Phys. Rev. B 47, 1651 (1993).

[2] R. Resta, Rev. Mod. Phys. 66, 899 (1994).

[3] D. Vanderbilt and R. D. King-Smith, Phys. Rev. B 48, 4442 (1993).

[4] S. Nakamura, M. Senoh, S. I. Nagahama, N. Iwasa, T. Yamada, T. Matsushita, Y. Sugimoto, and H. Kiyoku, Appl. Phys. Lett. 70, 1417 (1997).

[5] H. Sakai, T. Takeuchi, S. Sota, M. Katsuragawa, M. Komori, H. Amano,and I. Akasaki, J. Cryst. Growth 189/190, 831 (1998).

[6] Y. F. Yu, B. P. Keller, P. Fini, S. Keller, T. J. Jenkins, L. T. Kehias, S. P. Denbaars, and U. K. Mishra, IEEE Electron Device Lett. 19, 50 (1998).

[7] W. Walukiewicz, H. E. Ruda, J. Lagowski, and H. C. Gatos, Phys. Rev. B 30, 4571 (1984).

[8] T. Ando, A. B. Fowler, and F. Stern, Rev. Mod. Phys. 54, 437 (1982).

[9] M. A. Khan, J. W. Yang, G. Simin, R. Gaska, M. S. Shur, and A. D. Bykhovski, Appl. Phys. Lett. 75, 2806 (1999).

[10] E. T. Yu, G. J. Sullivan, P. M. Asbeck, C. D. Wang, D. Qiao, and S. S. Lau, Appl. Phys. Lett. 71, 2794 (1997).

[11] L. Hsu and W. Walukiewicz, Phys. Rev. B 56, 1520 (1997).

[12] R. Oberhuber, G. Zandler, and P. Vogl, Appl. Phys. Lett. 73, 818 (1998).

[13] R. Stratton, J. Phys. Chem. Solids 23, 1011 (1962).

[14] B. K. Ridley, Quantum processes in semiconductors (Clanderon Press, Oxford, 1982), p.168.

[15] F. Bernardini, V. Fiorentini, and D. Vanderbilt, Phys. Rev. B 56, R10 024 (1997).

[16] D. K. Ferry and S. M. Goodnick, Transport in Nanostructures (Cambridge University Press, Cambridge, 1997), p.60-70.

[17] J. H. Davies, The Physics of Low Dimensional Semiconductors (Cambridge University Press, Cambridge, 1998), p.357.

[18] O. Ambacher, B. Foutz, J. Smart, J. R. Shealy, N. G. Weimann, K. Chu, M. Murphy, A. J. Sierakowski, W. J. Schaff, L. F. Eastman, R. Dimitrov, A. Mitchell, and M. Stutzmann, J. Appl. Phys. 87, 334 (2000).

[19] I. P. Smorchkova, C. R. Elsass, J. P. Ibbetson, R. Vetury, B. Heying, P. Fini, E. Haus, S. P. Denbaars, J. S. Speck, and U. K. Mishra, J. Appl. Phys. 86, 4520 (1999).

[20] N. W. Ashcroft and N. D. Mermin, Solid State Physics (Saunders College Publishing, Orlando, 1976), p.555.

[21] L. N. Pfeiffer, K. W. West, H. L. Stormer, and K. W. Baldwin, Appl. Phys. Lett. 55, 1888 (1989).

[22] Y. Zhang and J. Singh, J. Appl. Phys. 85, 587 (1999).

[23] I. P. Smorchkova (unpublished).

[24] D. Jena, A. C. Gossard, and U. K. Mishra, Appl. Phys. Lett. 76, 1707 (2000).

[25] T. S. Zheleva, O. H. Nam, M. D. Bremser, and R. F. Davis, Appl. Phys. Lett. 71, 2472 (1997). 


\section{FIGURES}

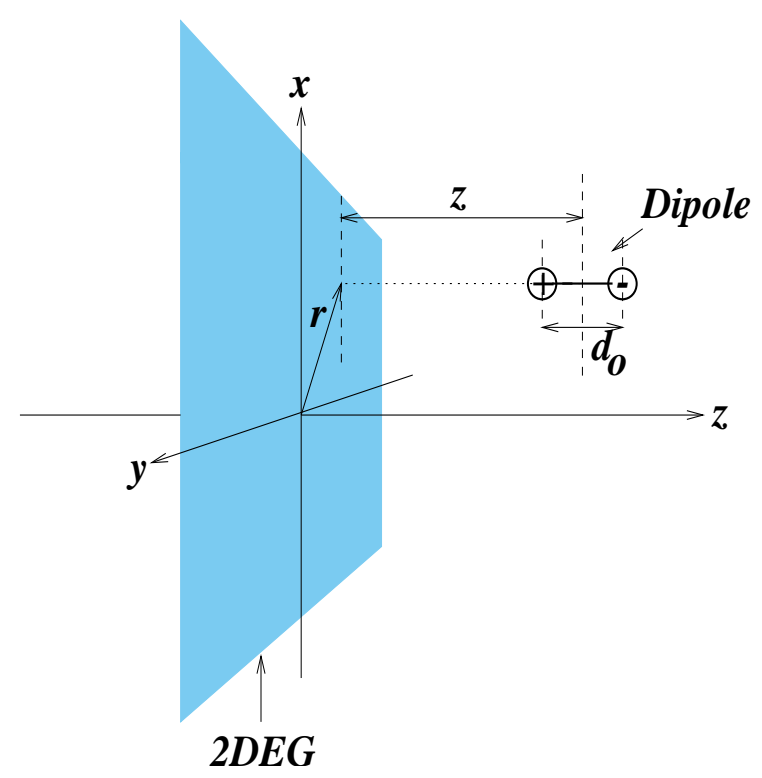

FIG. 1. The location of the dipole with respect to the 2DEG is shown. The dipole axis is taken to be perpendicular to the plane of the 2DEG, keeping with the direction of the polarization field in the AlGaN barrier of AlGaN/GaN HEMTs. The distances used in the text in the derivation of the scattering rate are defined. 


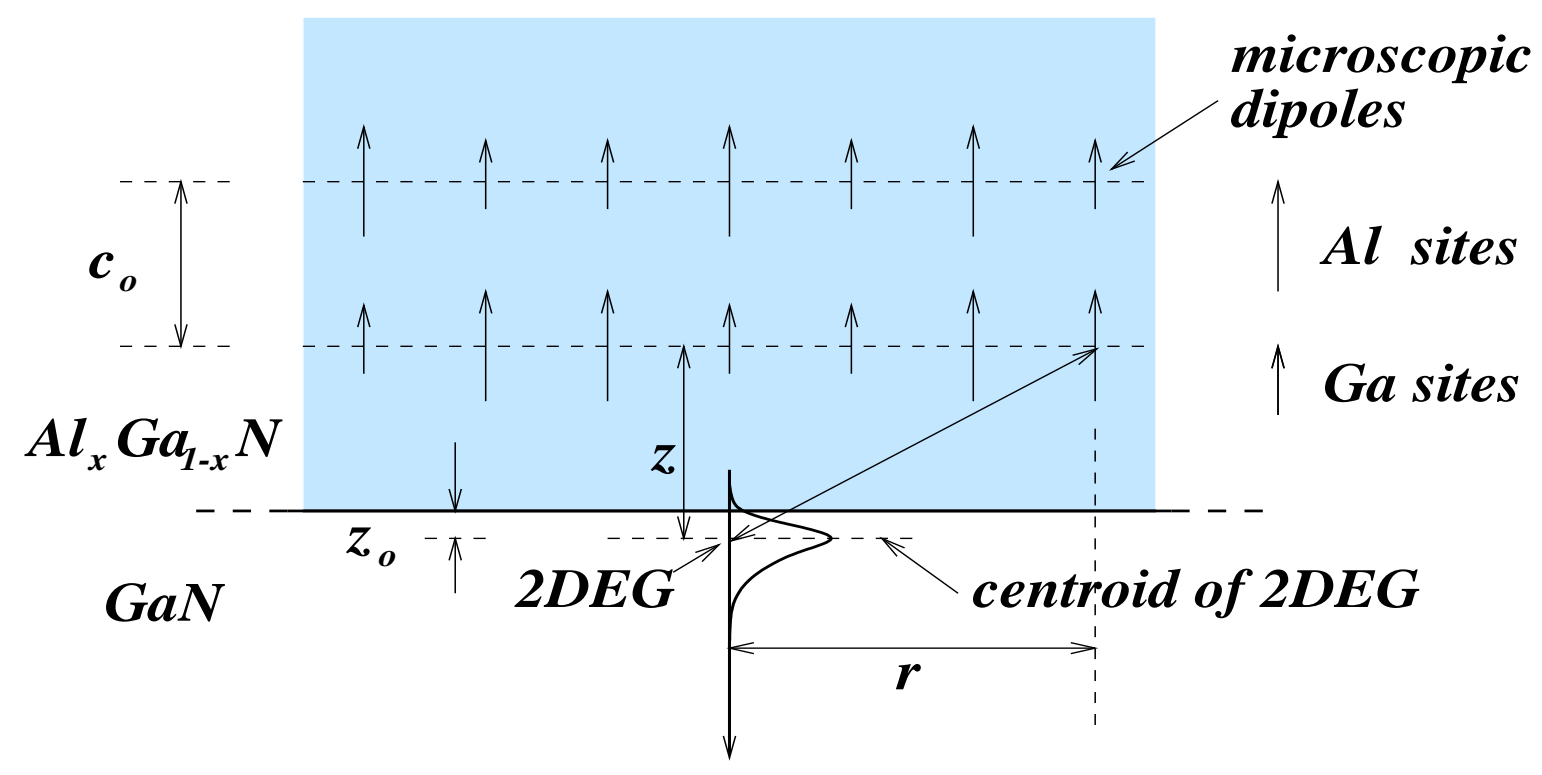

FIG. 2. The distribution of the dipoles in the AlGaN barrier is shown. Every $\mathrm{Al}(\mathrm{Ga})$ plane has dipoles in each primitive cell. The dipole moment at $\mathrm{Al}$ sites is higher than that at the Ga sites owing to the higher spontaneous polarization and piezoelectric constants in AlN than in GaN. This fluctuation leads to a random distribution of dipole moments which leads to scattering of the electrons in the 2DEG. The 2DEG is assumed to be located entirely at the centroid of the quasi-2DEG distribution for simplicity. 

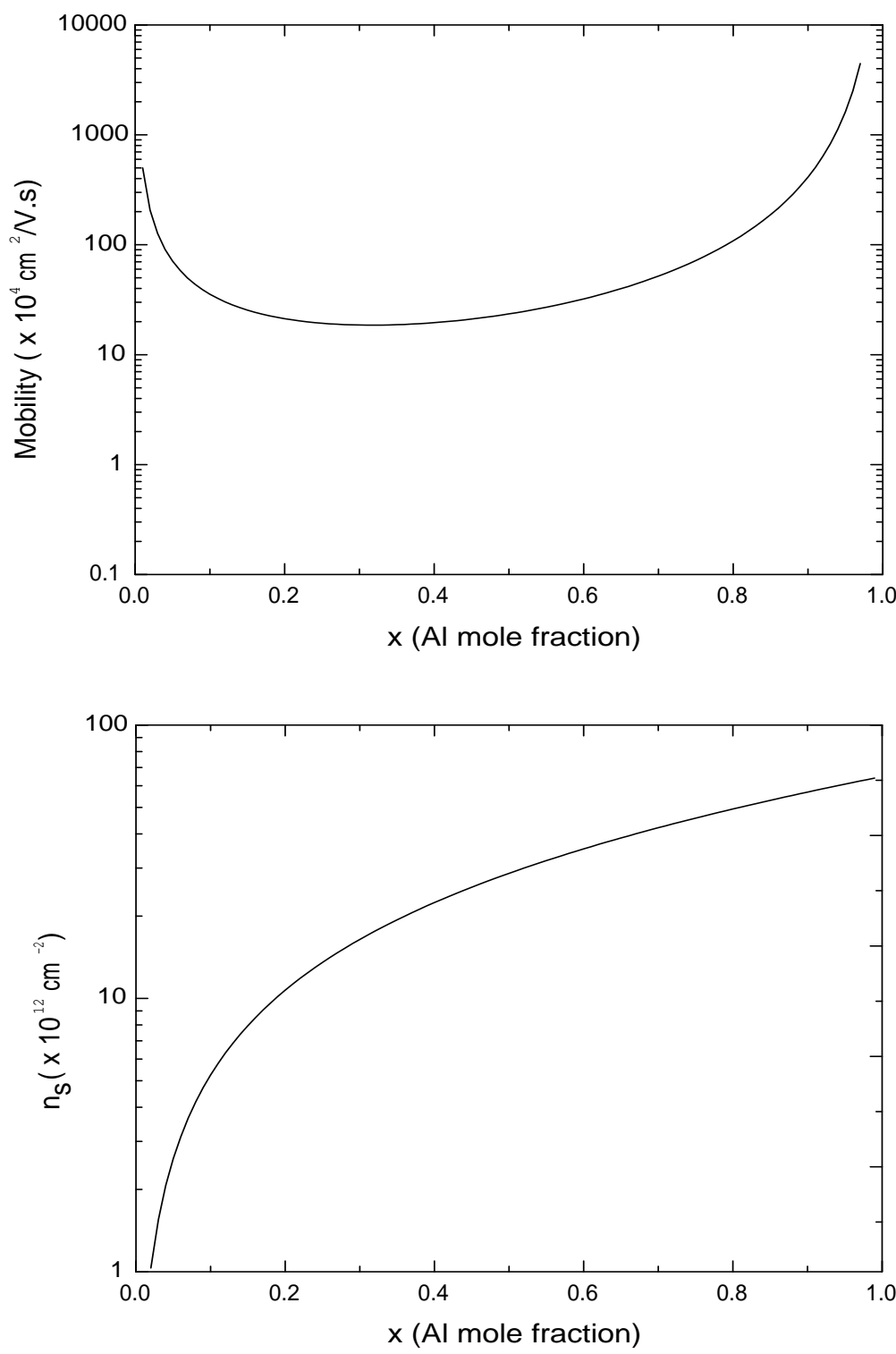

FIG. 3. Mobility of electrons in the 2DEG inhibited by dipole scattering alone is plotted as a function of alloy composition. Sheet density of carriers also changes with alloy composition, and is shown in the lower half. Dipole scattering dominates at alloy compositions in the $x=0.2-0.5$ range. As the binary nature of the alloy increases, dipole scattering reduces, leading to higher mobilities. 


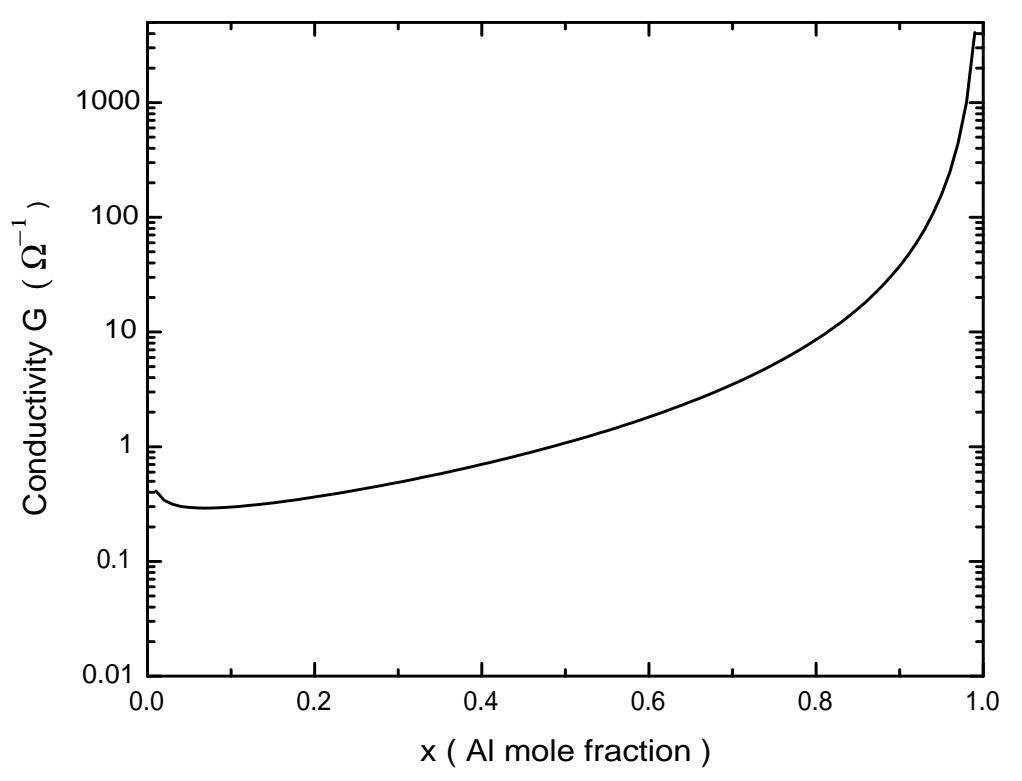

FIG. 4. Two-dimensional sheet conductivity $G=e \mu_{\text {dipole }}^{2 D} n_{s}$, inhibited by dipole scattering alone is plotted as a function of alloy composition. 


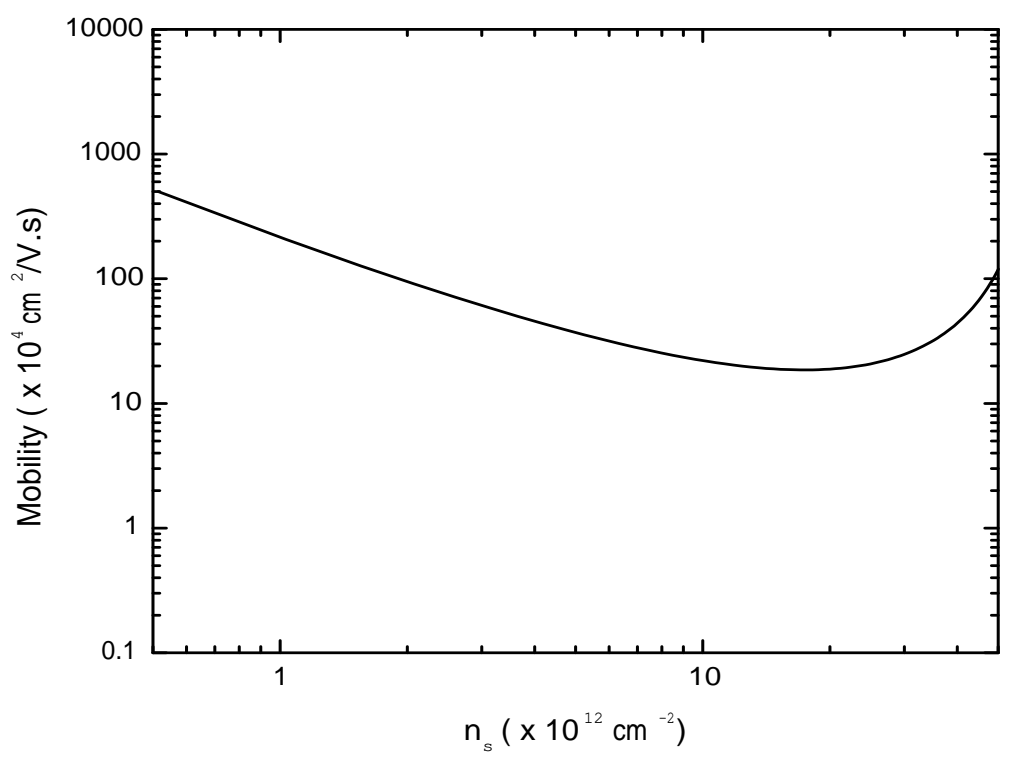

FIG. 5. Mobility limited by dipole scattering alone is plotted as a function of sheet density of carriers. The mobility reaches a minimum at 2DEG sheet density of $n_{s} \approx 2 \times 10^{13} / \mathrm{cm}^{2}$. 